\title{
Daphnetin protects oxidative stress-induced neuronal apoptosis via regulation of MAPK signaling and HSP70 expression
}

\author{
ZHILIN QI ${ }^{1}$, SHIMEI QI ${ }^{1}$, LIN GUI $^{2}$, LEI SHEN $^{3}$ and ZUNYONG FENG ${ }^{4}$ \\ Departments of ${ }^{1}$ Biochemistry and ${ }^{2}$ Microbiology and Immunology, Wannan Medical College, Wuhu, \\ Anhui 241002; ${ }^{3}$ Jiangsu Key Laboratory for Molecular and Medical Biotechnology, \\ College of Life Science, Nanjing Normal University, Nanjing, Jiangsu 210046; \\ ${ }^{4}$ Anhui Province Key Laboratory of Active Biological Macromolecules, \\ Wannan Medical College, Wuhu, Anhui 241002, P.R. China
}

Received May 7, 2015; Accepted June 16, 2016

DOI: $10.3892 / \mathrm{ol} .2016 .4849$

\begin{abstract}
Neurodegenerative disorders are characterized by progressive degeneration and loss of neurons in the brain. Oxidative stress is implicated in the pathogenesis of neurological disorders, although the pathological mechanism remains unelucidated. Daphnetin, an active ingredient extracted from Changbai daphne (Daphne Korean Nakai), exhibits various pharmacological effects, including anti-inflammatory, anti-oxidative and anti-tumor effects. However, the neuroprotective effects, as well as the specific mechanisms of daphnetin, remain unclear. Neuronal-like rat pheochromocytoma PC12 cells were pretreated with daphnetin for $2 \mathrm{~h}$, then treated with or without $\mathrm{H}_{2} \mathrm{O}_{2}$ for various times. Cell morphology was detected using an inverted microscope, the apoptotic ratio was determined by Annexin V fluorescein isothiocyanate/ propidium iodide assay, nuclear morphology was observed and photographed using a fluorescence microscope following 4',6-diamidino-2-phenylindole staining. The levels of procaspase 3 , cleavage of poly ADP-ribose polymerase and caspase 3 were detected by western blotting. In addition, the activation of mitogen-activated protein kinase (MAPK) signal pathway and the expression of HSP70 were detected by western blotting. The present study demonstrated that daphnetin attenuated hydrogen peroxide $\left(\mathrm{H}_{2} \mathrm{O}_{2}\right)$-induced apoptosis in a concentration-dependent manner, reduced the cleavage of poly ADP ribose polymerase and caspase 3, and inhibited the phosphorylation of p38 MAPK and c-Jun N-terminal kinases (JNK) in $\mathrm{H}_{2} \mathrm{O}_{2}$-induced $\mathrm{PC} 12$ cells. In addition, daphnetin induced the
\end{abstract}

Correspondence to: Dr Zhilin Qi or Dr Shimei Qi, Department of Biochemistry, Wannan Medical College, 22 Wenchang West Road, Wuhu, Anhui 241002, P.R. China

E-mail: 422627721@qq.com

E-mail: juliaqi@163.com

Abbreviations: DAPI, 4',6-diamidino-2-phenylindole; $\mathrm{H}_{2} \mathrm{O}_{2}$, hydrogen peroxide; HSP, heat shock protein

Key words: daphnetin, oxidative stress, apoptosis, MAPK, HSP70 expression of HSP70 in a dose- and time-dependent manner, and daphnetin-induced HSP70 expression was reduced by extracellular signal-regulated kinase (ERK) 1/2 inhibitor U0126 in PC1 2 cells. Therefore, the present results indicate that daphnetin protects PC12 cells against oxidative stress injury by regulating p38 MAPK and JNK signaling and increasing the expression of HSP70 via ERK signaling. This suggests that daphnetin may have the potential to treat certain neurodegenerative diseases. The present results not only provide insight into the potential use of daphnetin in $\mathrm{H}_{2} \mathrm{O}_{2}$-induced $\mathrm{PC} 12$ cell apoptosis, but also highlight the potential role of HSP70 in neuroprotection.

\section{Introduction}

Oxidative stress is associated with the pathogenesis of neurological disorders, including dysautonomia, Alzheimer's disease and Parkinson's disease $(1,2)$. Hydrogen peroxide $\left(\mathrm{H}_{2} \mathrm{O}_{2}\right)$, which is one of the major reactive oxygen species, is considered as a major cause of neuronal cell death (3). Therefore, pharmacological approaches for intervening in oxidative stress may be potential therapeutic strategies for neurodegenerative disorders (4).

Natural antioxidants with neuroprotective potential are being considered as a promising approach to prevent or slow the effects of neurological illness, due to their low toxicity and absence of clear side effects. It has been reported that numerous natural antioxidants, including resveratrol, celastrol and salidroside, may protect neurons from oxidative stress injury (5-7).

Daphnetin (7,8-dihydroxycoumarin), an active ingredient extracted from Changbai daphne (Daphne Korean Nakai), exhibits various pharmacological effects, including anti-inflammatory, anti-oxidative and anti-tumor effects $(8,9)$. However, whether daphnetin exerts neuroprotection against $\mathrm{H}_{2} \mathrm{O}_{2}$-induced neuronal-like rat pheochromocytoma PC12 cell apoptosis, and the mechanisms responsible for this effect, remains unclear.

Inducible heat shock protein (HSP) 70, a member of the HSP superfamily, is an important protective protein induced by various stimuli that prevents cell apoptosis $(10,11)$. A previous study has suggested that HSP70 is protective in neurodegenerative diseases, including Parkinson's disease, through its chaperone and direct antiapoptotic role (11). It has also been 
reported that natural antioxidants, including celastrol, protect nerve cell damage by inducing the expression of HSP70 (12).

The present study investigated the activity of daphnetin in neuronal apoptosis and the underlying mechanisms of this effect. The present study demonstrated that daphnetin dose-dependently attenuated $\mathrm{H}_{2} \mathrm{O}_{2}$-induced $\mathrm{PC} 12$ cell apoptosis via suppression of p38 and c-Jun N-terminal kinases (JNK) phosphorylation. In addition, the present study revealed that HSP70 expression was elevated in daphnetin-treated PC12 cells, and HSP70 expression was regulated by extracellular signal-regulated kinase (ERK) signaling. Overall, the present study concluded that daphnetin attenuates p38 and JNK activation and upregulates HSP70 expression in $\mathrm{H}_{2} \mathrm{O}_{2}$-treated PC12 cells. These two mechanisms reduce $\mathrm{H}_{2} \mathrm{O}_{2}$-induced $\mathrm{PC} 12$ apoptosis, and are protective in oxidative stress-induced neuronal injury.

\section{Materials and methods}

Antibodies and reagents. Daphnetin (purity >98\%) was obtained from Sigma-Aldrich (St. Louis, MO, USA), and the ERK inhibitor U0126 was purchased from Cell Signaling Technology, Inc. (Danvers, MA, USA). $\mathrm{H}_{2} \mathrm{O}_{2}(30 \%)$ was purchased from Beyotime Institute of Biotechnology (Shanghai, China). Rabbit monoclonal antibodies against $\beta$-actin (catalog no., 4970S), Akt, phospho (p)-Akt (Ser 473; catalog no., 9272S), p38 mitogen-activated protein kinase (MAPK; catalog no., 8690S), p-p38 MAPK (Thr180/Tyr182; catalog no., 4511S), ERK (catalog no., 4695S), p-ERK (Thr202/Tyr204; catalog no., 4376S), JNK/stress-activated protein kinase (SAPK; catalog no., 9258S), p-JNK/SAPK (Thr183/Tyr185; catalog no., 4668S), poly ADP-ribose polymerase (PARP; catalog no., 9532S), cleaved-caspase 3 (catalog no., 9664S; 1:500), pro-caspase 3 (catalog no., 9665S) and HSP70 (catalog no., 4872S) were all purchased from Cell Signaling Technology, Inc and used at 1:1,000 dilution, unless otherwise specified. Rabbit polyclonal antibody against glyceraldehyde 3-phosphate dehydrogenase (catalog no., AP0063; 1:1,000) was purchased from Bioworld Technology, Inc. (St. Louis Park, MN, USA). Secondary antibodies coupled to IRDye800 fluorophore (catalog no., 926-32211; dilution, 1:5,000) for use with the Odyssey ${ }^{\circledR}$ Infrared Imaging System (LI-COR Biosciences, Lincoln, NE, USA) were purchased from LI-COR Biosciences.

Cell culture. Rat pheochromocytoma PC12 cells were obtained from the American Type Culture Collection (Manassas, VA, USA) and cultured in Dulbecco's modified Eagle's medium (Invitrogen $^{\mathrm{TM}}$; Thermo Fisher, Scientific, Inc., Waltham, MA, USA) supplemented with $10 \%$ (v/v) heat-inactivated horse serum, $5 \%$ fetal bovine serum and $1 \%$ antibiotics (100 U/ml penicillin and $100 \mu \mathrm{g} / \mathrm{ml}$ streptomycin) (Hyclone ${ }^{\mathrm{TM}}$; GE Healthcare Life Sciences, Logan, UT, USA) at $37^{\circ} \mathrm{C}$ in $5 \%$ $\mathrm{CO}_{2} / 95 \%$ air humidified atmosphere. Culture medium was changed every 2-3 days.

Cell viability assay. Cell viability was detected using Cell Counting Kit-8 (CCK-8; KeyGen Biotech Corp., Ltd., Nanjing, China). Briefly, PC12 cells were plated onto 96-well plates at a density of $2 \times 10^{4}$ cells per well $24 \mathrm{~h}$ prior to treatment. Cells were treated with various concentrations of daphnetin $(0,2.5$, 5.0, 10.0 and $20.0 \mu \mathrm{M}$ ) for $2 \mathrm{~h}$, then stimulated with $\mathrm{H}_{2} \mathrm{O}_{2}$
$(200 \mu \mathrm{M})$ for $24 \mathrm{~h}$, followed by incubation with $10 \mu \mathrm{l} \mathrm{CCK}-8$ working solution at $37 \%$ for $4 \mathrm{~h}$. The absorbance of each well at $450 \mathrm{~nm}$ was measured using a Multiskan ${ }^{\mathrm{TM}}$ GO Microplate Spectrophotometer (Thermo Fisher Scientific, Inc.). Three repeats were performed for each of the different treatments.

Annexin V-fluorescein isothiocyanate (FITC)/propidium iodide (PI) assay. The apoptotic ratio was analyzed using Annexin-V/PI Double Staining Assay (KeyGen Biotech Corp., Ltd.), according to the manufacturer's protocol. Briefly, the cells were washed, trypsinized and resuspended with $500 \mu \mathrm{l}$ binding buffer, then stained with Annexin V-FITC (5 $\mu \mathrm{l})$ and PI $(5 \mu \mathrm{l})$. The stained cells were visualized directly using a Guava EasyCyte ${ }^{\mathrm{TM}}$ System (EMD Millipore, Billerica, MA, USA), and the data were analyzed using Guava TUNEL ExpressPro version 8.0 software (EMD Millipore).

Western blotting. Cells were rinsed twice with ice-cold phosphate-buffered saline (PBS) and lysed on ice in a lysis buffer containing $20 \mathrm{mM}$ Tris ( $\mathrm{pH} 7.5$ ), $2 \mathrm{mM}$ EDTA, $135 \mathrm{mM} \mathrm{NaCl}, 2 \mathrm{mM}$ DTT, $2 \mathrm{mM}$ sodium pyrophosphate, $25 \mathrm{mM} \beta$-glycerophosphate, $10 \%$ glycerol, $1 \%$ Triton $\mathrm{X}-100$, $1 \mathrm{mM} \mathrm{Na}{ }_{3} \mathrm{VO}_{4}, 10 \mathrm{mM} \mathrm{NaF}, 10 \mu \mathrm{g} / \mathrm{ml}$ leupeptin, $10 \mu \mathrm{g} / \mathrm{ml}$ aprotinin and $1 \mathrm{mM}$ PMSF, supplemented with a complete protease inhibitor cocktail (Roche Diagnostics, Indianapolis, IN, USA) for $30 \mathrm{~min}$. Lysates were centrifuged at $12,500 \mathrm{x} \mathrm{g}$ for $15 \mathrm{~min}$ at $4^{\circ} \mathrm{C}$. Equal amounts of proteins were subjected to $12 \%$ SDS-PAGE, and then transferred onto nitrocellulose membranes (GE Healthcare Life Sciences, Chalfont, UK). The membrane was blocked with 5\% skimmed milk for $1 \mathrm{~h}$ at room temperature, washed with Tris-buffered saline with Tween 20 three times, then incubated with the indicated primary antibodies at $4^{\circ} \mathrm{C}$ overnight. Subsequently, the membranes were incubated with secondary antibodies for $1 \mathrm{~h}$ at room temperature. The antibody-antigen complexes were visualized by the Odyssey Infrared Imaging System using IRDye800 fluorophore-conjugated antibodies (LI-CORBiosciences).

Morphological observation. PC12 cells were pretreated with various concentrations of daphnetin $(5,10$ and $20 \mu \mathrm{M})$ for $2 \mathrm{~h}$, then stimulated with $\mathrm{H}_{2} \mathrm{O}_{2}(200 \mu \mathrm{M})$ for $24 \mathrm{~h}$. Subsequently, cell morphology was observed using an inverted microscope (DP72; Olympus Corporation, Tokyo, Japan).

Immunofluorescence microscopy and 4',6-diamidino-2-phenylindole (DAPI) staining. Cells were pre-incubated with daphnetin for $2 \mathrm{~h}$, then stimulated with $\mathrm{H}_{2} \mathrm{O}_{2}$ for $24 \mathrm{~h}$. Cells were fixed with $4 \%$ paraformaldehyde for $20 \mathrm{~min}$ at room temperature, permeabilized in $0.2 \%$ Triton $\mathrm{X}-100$ and then incubated with DAPI $(1 \mu \mathrm{g} / \mathrm{ml})$ for $5 \mathrm{~min}$ in the dark. Subsequent to washing with PBS, nuclear morphology was observed and photographed using a fluorescence microscope (DP72; Olympus Corporation).

Statistical analysis. Data are expressed as the mean \pm standard deviation. One-way analysis of variance was used to determine the significance of the difference between two groups. $\mathrm{P}<0.05$ was considered to indicate a statistically significant difference. Statistical analysis was performed using SPSS version 17.0 software (SPSS, Inc., Chicago, IL, USA). 

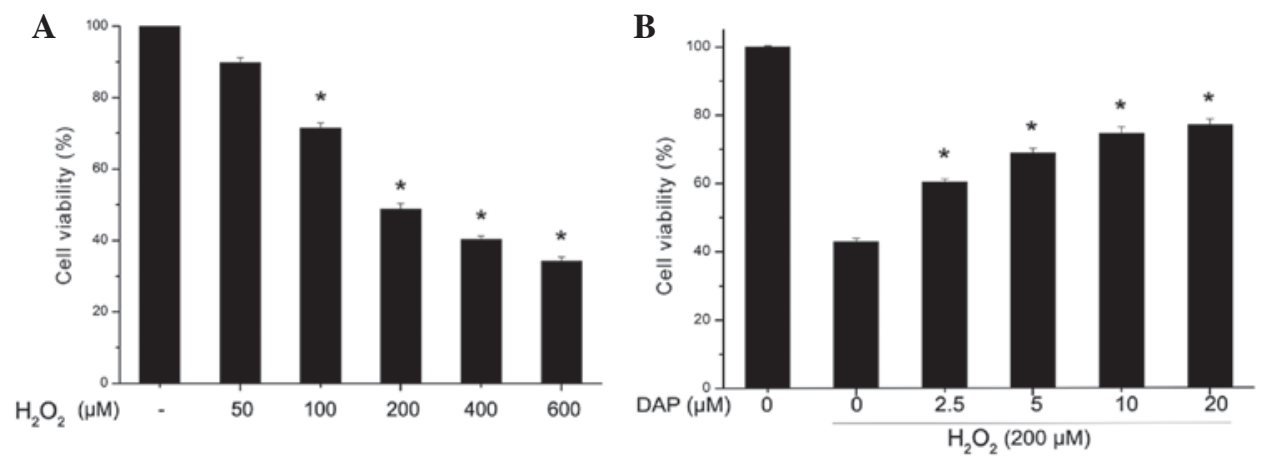

Figure 1. DAP enhanced $\mathrm{H}_{2} \mathrm{O}_{2}$-induced cell viability. (A) Rat pheochromocytoma PC12 cells were treated with various concentrations of $\mathrm{H}_{2} \mathrm{O}_{2}$ for $24 \mathrm{~h}$, (B) $\mathrm{PC} 12$ cells were pretreated with various doses of DAP for $2 \mathrm{~h}$, then stimulated with $\mathrm{H}_{2} \mathrm{O}_{2}(200 \mu \mathrm{M})$ for $24 \mathrm{~h}$. Cell viability was determined by Cell Counting Kit-8. Experiments were independently repeated three times. (A) " $\mathrm{P}<0.05$ vs. control; (B) " $\mathrm{P}<0.05$ vs. $\mathrm{H}_{2} \mathrm{O}_{2}$-treated group. $\mathrm{H}_{2} \mathrm{O}_{2}$, hydrogen peroxide; DAP, daphnetin.

\section{Results}

Effect of daphnetin on $\mathrm{H}_{2} \mathrm{O}_{2}$-induced cell viability. $\mathrm{PC} 12$ cells were treated with various concentrations of $\mathrm{H}_{2} \mathrm{O}_{2}(50$, $100,200,400$ and $600 \mu \mathrm{M}$ ) for $24 \mathrm{~h}$, and subsequently cell viability was determined using CCK-8. As shown in Fig. 1A, $\mathrm{H}_{2} \mathrm{O}_{2}$ reduced cell viability in a dose-dependent manner ( $\mathrm{P}<0.05$ vs. control). For the cells treated with $200 \mu \mathrm{M} \mathrm{H}_{2} \mathrm{O}_{2}$, the cell viability was $48.75 \pm 1.63 \%$. Since, the cell viability was reduced by $\sim 50 \%$ with $200 \mu \mathrm{M} \mathrm{H}_{2} \mathrm{O}_{2}$ at $24 \mathrm{~h}$, in subsequent experiments these variables were selected as the standard dose and time point for the induction of PC12 cell apoptosis. In $\mathrm{PC} 12$ cells treated with various doses of daphnetin and then stimulated with $200 \mu \mathrm{M} \mathrm{H}_{2} \mathrm{O}_{2}$, cell viability was increased in a daphnetin dose-dependent manner $\left(\mathrm{P}<0.05\right.$ vs. $\mathrm{H}_{2} \mathrm{O}_{2}$-treated group; Fig. 1B).

Daphnetin attenuated $\mathrm{H}_{2} \mathrm{O}_{2}$-induced $\mathrm{PCl} 2$ cell apoptosis. Observation of cell morphology revealed that untreated PC12 cells exhibited typical long fusiform-like morphology. By contrast, $\mathrm{H}_{2} \mathrm{O}_{2}$-treated $(200 \mu \mathrm{M} ; 24$ h) PC12 cells were shrunken, scattered and floating. Pretreating cells with daphnetin significantly reversed the apoptotic morphological alterations observed with $\mathrm{H}_{2} \mathrm{O}_{2}$-treated cells (Fig. 2A). To further determine the role of daphnetin in suppressing $\mathrm{H}_{2} \mathrm{O}_{2}$-induced $\mathrm{PC} 12$ cell apoptosis, the apoptotic ratio was determined by Annexin V-FITC/PI assay. As shown in Fig. 2B, cells exposed to $\mathrm{H}_{2} \mathrm{O}_{2}$ for $24 \mathrm{~h}$ had an apoptotic ratio of $55.6 \%$. However, the number of apoptotic cells, when cells were pretreated with various concentrations of daphnetin, was significantly reduced; the apoptotic ratio was $34.6,31.3$, $26.6 \%$, for 5,10 and $20 \mu \mathrm{M}$ daphnetin, respectively.

The activation of apoptosis-associated proteins was detected using western blotting to confirm the occurrence of apoptosis. Daphnetin pretreated cells clearly attenuated the $\mathrm{H}_{2} \mathrm{O}_{2}$-induced cleavage of PARP and caspase 3 , and enhanced the level of pro-caspase 3 (Fig. 2C) compared with cells not pretreated with daphnetin. DAPI staining was used to determine the apoptotic status of the PC12 cells. Staining with DAPI revealed that the round nuclei of normal cells was homogeneous, and when exposed to $200 \mu \mathrm{M} \mathrm{H}_{2} \mathrm{O}_{2}$ for $24 \mathrm{~h}$ cells underwent nuclear condensation and fragmentation. However, these alterations in nuclear morphology were significantly attenuated by pretreatment with daphnetin (Fig. 2D). Overall, these results suggest that daphnetin has a protective effect in $\mathrm{H}_{2} \mathrm{O}_{2}$-induced $\mathrm{PC} 12$ cell apoptosis.

Daphnetin reduced the activation of p38 MAPK and JNK induced by $\mathrm{H}_{2} \mathrm{O}_{2}$. To determine the mechanism by which daphnetin inhibits $\mathrm{H}_{2} \mathrm{O}_{2}$-induced apoptosis, the phosphorylation levels of MAPKs in PC12 cells were investigated. PC12 cells were stimulated with $200 \mu \mathrm{M} \mathrm{H}_{2} \mathrm{O}_{2}$ for various times, and the phosphorylation levels of p38, ERK and JNK were detected by western blotting. The results showed that stimulation of PC12 cells with $\mathrm{H}_{2} \mathrm{O}_{2}$ resulted in an increase in phosphorylation of JNK, p38 and ERK (Fig. 3A). The enhanced phosphorylation of JNK and p38 was significantly attenuated by daphnetin pretreatment in a dose-dependent manner (Fig. 3B). However in the presence of daphnetin, increased ERK phosphorylation observed with $\mathrm{H}_{2} \mathrm{O}_{2}$ stimulation was not attenuated. In addition, with daphnetin stimulation, phosphorylation of ERK was clearly elevated, but ERK activation had no additive effect on daphnetin and $\mathrm{H}_{2} \mathrm{O}_{2}$ stimulation (Fig. 3B).

Daphnetin induced HSP70 expression in PC12 cells. In order to determine other mediators in daphnetin neuroprotection, HSP70, a well-known chaperone with cytoprotective effects, was investigated. PC12 cells were stimulated with daphnetin, and total cellular protein was extracted and subjected to western blotting with an anti-HSP70 antibody. The present results revealed that the level of HSP70 was increased upon daphnetin stimulation in a dose- and time-dependent manner (Fig. 4A and B). The level of HSP70 peaked at $12 \mathrm{~h}$.

ERK signaling is involved in daphnetin-induced HSP7O expression. The phosphorylation of upstream kinases was examined to investigate the possible signal transduction pathways involved in daphnetin-induced HSP70 expression. PC12 cells were treated with daphnetin $(20 \mu \mathrm{M})$ for various times and the phosphorylation level of ERK and Akt was detected by western blotting. As shown in Fig. 5A, the activation of ERK was enhanced in a time-dependent manner following daphnetin stimulation. ERK activation peaked at $12 \mathrm{~h}$, which is consistent with the expression of HSP70. By contrast, Akt phosphorylation was not significantly different compared with unstimulated PC12 cells. Overall, these observations suggested that ERK signaling activation may be involved in daphnetin-induced HSP70 expression. 
A
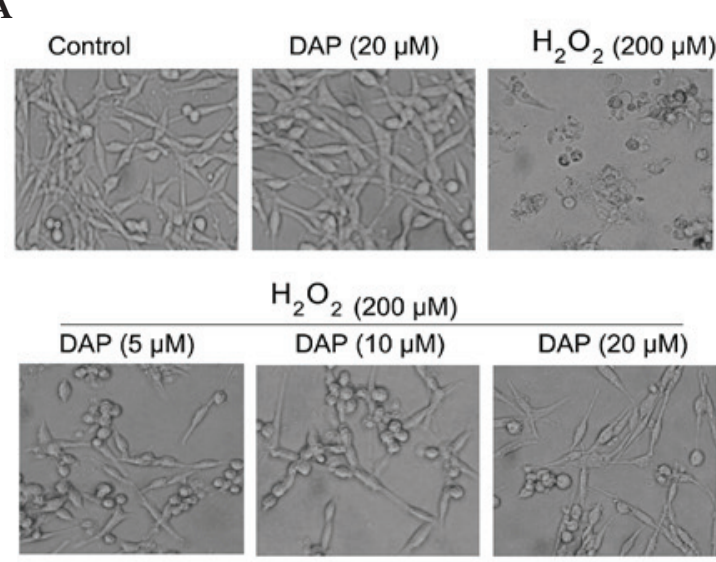

C

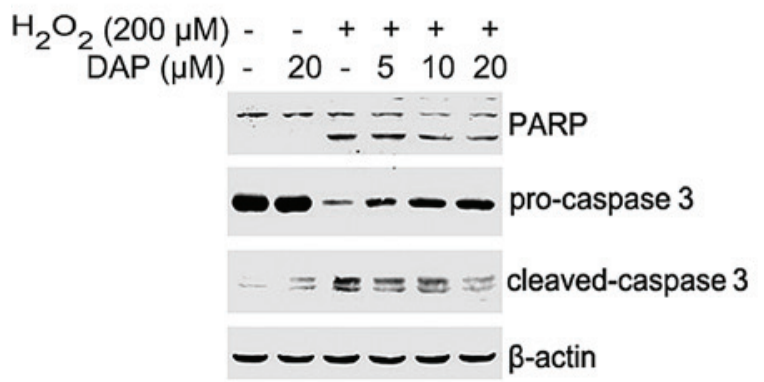

B

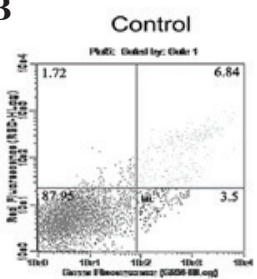

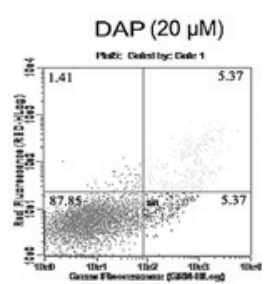

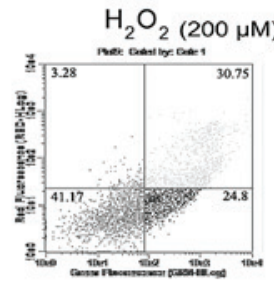

$\mathrm{H}_{2} \mathrm{O}_{2}(200 \mu \mathrm{M})$
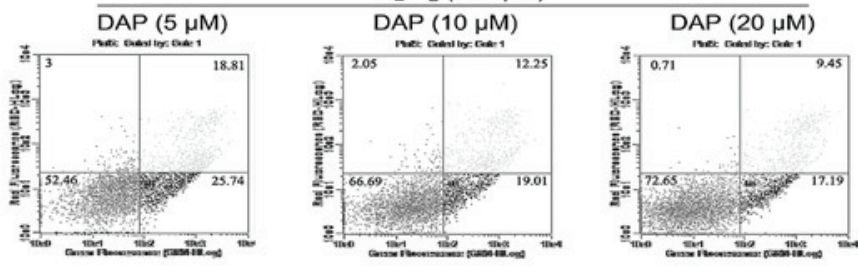

D
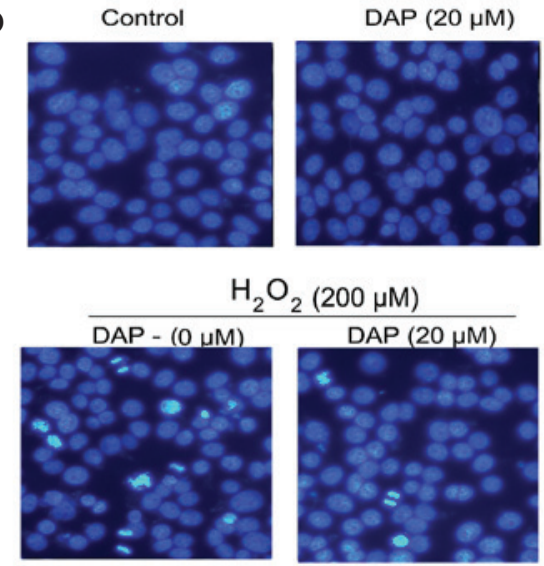

Figure 2. DAP attenuated $\mathrm{H}_{2} \mathrm{O}_{2}$-induced apoptosis in rat pheochromocytoma PC12 cells. PC12 cells were pretreated with various concentrations of daphnetin for $2 \mathrm{~h}$, then stimulated with $\mathrm{H}_{2} \mathrm{O}_{2}(200 \mu \mathrm{M})$ for $24 \mathrm{~h}$. (A) Cell morphology was detected using an inverted microscope. (B) The apoptotic ratio was determined by Annexin V-FITC/PI assay. (C) Cleavage of PARP $(89 / 116 \mathrm{kDa})$ and caspase $3(17 / 19 \mathrm{kDa})$, and the level of pro-caspase $3(35 \mathrm{kDa})$ were detected by western blotting ( $\beta$-actin, $45 \mathrm{kDa}$ ). (D) Cells were stained with 4',6-diamidino-2-phenylindole, and nuclear morphology was observed and photographed using a fluorescence microscope. $\mathrm{H}_{2} \mathrm{O}_{2}$, hydrogen peroxide; DAP, daphnetin; FITC, fluorescein isothiocyanate; PI, propidium iodide; PARP, poly ADP-ribose polymerase.

A

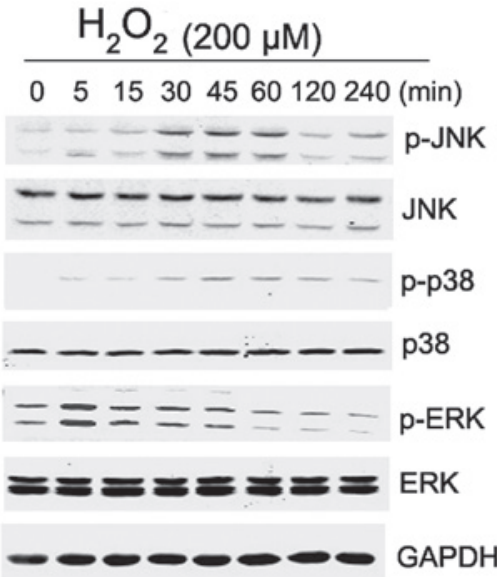

B

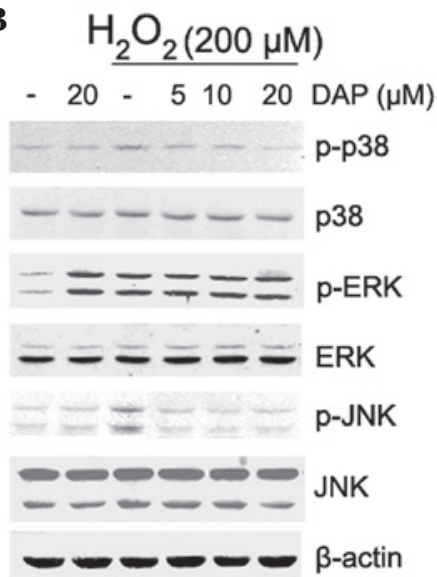

Figure 3. DAP reduced $\mathrm{H}_{2} \mathrm{O}_{2}$-induced p38 mitogen-activated protein kinase and JNK activation. (A and B) The phosphorylation level of p38 (43 kDa), ERK $(42 / 44 \mathrm{kDa})$ and JNK (46/54 kDa) was detected by western blotting in (A) rat pheochromocytoma PC12 cells stimulated with $200 \mu \mathrm{M} \mathrm{H}_{2} \mathrm{O}_{2}$ for various times and (B) PC12 cells pretreated with DAP $(5,10,20 \mu \mathrm{M})$ for $2 \mathrm{~h}$, then stimulated with $\mathrm{H}_{2} \mathrm{O}_{2}(200 \mu \mathrm{M})$ for 30 min. Equal protein loading was confirmed by GAPDH (37 kDa) and $\beta$-actin $(45 \mathrm{kDa}) . \mathrm{H}_{2} \mathrm{O}_{2}$, hydrogen peroxide; DAP, daphnetin; JNK, c-Jun N-terminal kinases; ERK, extracellular signal-regulated kinase; p-phospho; GAPDH, glyceraldehyde 3-phosphate dehydrogenase.

To test this hypothesis, U0126, a specific inhibitor of ERK, was used to verify the activation of ERK in daphnetin-induced HSP70 expression. PC12 cells were pretreated with $20 \mu \mathrm{M}$
U0126 for $2 \mathrm{~h}$, and then exposed to $20 \mu \mathrm{M}$ daphnetin for a further $12 \mathrm{~h}$, and the expression of HSP70 and phosphorylated ERK were detected using western blotting. As shown in Fig. 5B, 

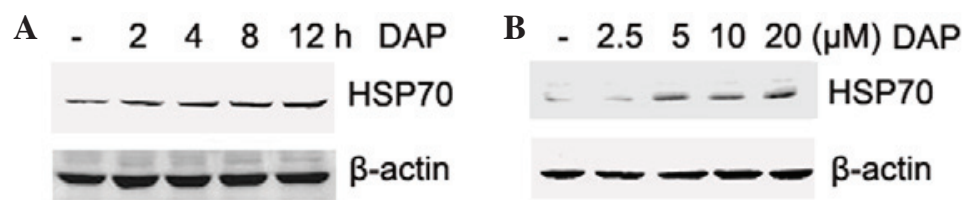

Figure 4. DAP induced HSP70 expression in rat pheochromocytoma PC12 cells. (A and B) PC12 cells were stimulated with (A) $20 \mu \mathrm{M}$ DAP for $2,4,8,12$ h or (B) various concentrations of DAP for $12 \mathrm{~h}$. Total cellular protein were extracted and subjected to western blotting probed with an anti-HSP70 (70 kDa) antibody. Equal protein loading was confirmed by $\beta$-actin (45 kDa). DAP, daphnetin; HSP, heat shock protein.

A

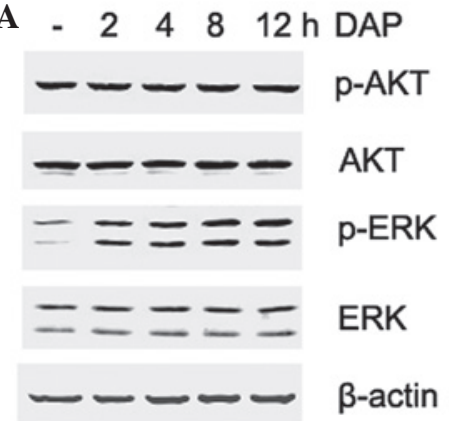

B

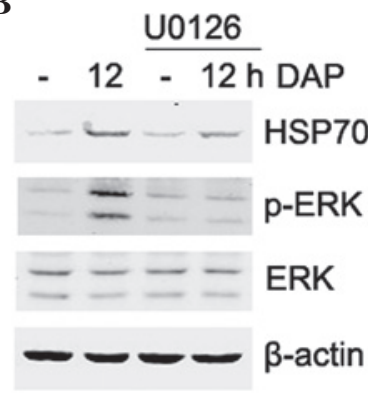

Figure 5. ERK signaling is involved in DAP-induced HSP70 expression. (A) Rat pheochromocytoma PC12 cells were stimulated with $20 \mu$ M daphnetin for 2, 4, 8 and $12 \mathrm{~h}$, and total cellular protein was extracted and subjected to western blotting with p-Akt (60 kDa), Akt (60 kDa), p-ERK (42/44 kDa) and ERK $(42 / 44 \mathrm{kDa}$ ) antibodies. (B) PC12 cells were pretreated with U0126 for $2 \mathrm{~h}$, then treated with daphnetin for $12 \mathrm{~h}$. Cell lysates were prepared and subjected to western blotting using HSP70 (70 kDa), p-ERK and ERK antibodies. Equal protein loading was confirmed by $\beta$-actin (45 kDa). DAP, daphnetin; HSP, heat shock protein; ERK, extracellular signal-regulated kinase; p, phospho.

attenuation of ERK activation by U0126 clearly reduced the protein expression level of HSP70. Therefore, the present data suggest that HSP70 is involved in daphnetin-mediated cytoprotection via ERK signaling.

\section{Discussion}

Oxidative stress has been widely implicated in neuronal cell death, and is associated with a variety of chronic neurodegenerative diseases, including Parkinson's, Alzheimer's and Huntington's disease. $\mathrm{H}_{2} \mathrm{O}_{2}$ is a strong oxidant, which reduces cell viability and increases cell apoptosis (13). Currently, great effort is being made to identify potent natural antioxidants with neuroprotective potential, due to their low toxicity and absence of clear side effects. Daphnetin, a natural antioxidant, exhibits a variety of biological effects, including anti-inflammatory and antitumor effects (14). Recently, studies have demonstrated that daphnetin exhibits a neurotrophic effect on peripheral nerve regeneration by suppressing nuclear factor- $\kappa \mathrm{B}$ expression (15), provides a neuroprotective effect against glutamate-induced toxicity in immortalized mouse hippocampal HT22 cells and ischemic brain injury (16), and prevents excitotoxicity by inhibiting the $\mathrm{N}$-methyl $\mathrm{D}$-aspartate receptor subtype $2 \mathrm{~B}$ -containing receptors and the subsequent calcium overload in cultured cortical neurons (17). These studies suggest that daphnetin has a neuroprotective effect, which is consistent with the present findings. The present study confirmed that treating PC12 cells with $\mathrm{H}_{2} \mathrm{O}_{2}$ resulted in suppression of cell viability in a dose-dependent manner; however, pretreatment of these cells with various concentrations $(5,10,20 \mu \mathrm{M})$ of daphnetin significantly increased PC12 cell viability (Fig. 1). The present study further investigated whether daphnetin has protective effects against $\mathrm{H}_{2} \mathrm{O}_{2}$-induced nerve cell apoptosis. Cell morphology analysis revealed that untreated PC12 cells exhibited typical long fusiform-like morphology, while treatment with $\mathrm{H}_{2} \mathrm{O}_{2}$ $(200 \mu \mathrm{M})$ for $24 \mathrm{~h}$ resulted in shrunken, scattered and floating cells. However, pre-incubation with daphnetin clearly reversed the morphology induced by $\mathrm{H}_{2} \mathrm{O}_{2}$ (Fig. 2A). The cell apoptotic ratio was detected using flow cytometry, and the present data revealed that the number of apoptotic cells was significantly reduced in daphnetin-pretreated $\mathrm{PC} 12$ cells compared with cells stimulated with $\mathrm{H}_{2} \mathrm{O}_{2}$ (Fig. 2B). The present study also examined the activation of apoptotic proteins by immunoblotting, in order to confirm the occurrence of apoptosis. Upon $\mathrm{H}_{2} \mathrm{O}_{2}$ stimulation, cleavage of PARP and caspase 3 were clearly enhanced in PC12 cells; however, pretreatment with daphnetin significantly decreased the cleavage of PARP and caspase 3 in a concentration-dependent manner (Fig. 2C). DAPI staining of $\mathrm{H}_{2} \mathrm{O}_{2}$-treated cells revealed typical apoptotic morphology. However, pre-incubation with daphnetin clearly attenuated these morphological alterations (Fig. 2D). These results suggest that daphnetin exerts its neuroprotective effect via inhibition of PC12 cell apoptosis.

Certain studies have revealed that oxidative stress-induced toxicity is associated with intracellular signaling, including the activation of JNK and p38 signaling pathways $(7,13)$. In addition, it has been reported that the activation of ERK may protect neurons from oxidative stress-induced cell death (18). The present study hypothesized that daphnetin may protect PC12 cells against $\mathrm{H}_{2} \mathrm{O}_{2}$-induced apoptosis via the MAPK signaling pathway. Western blotting revealed that p38, ERK and JNK were phosphorylated upon $\mathrm{H}_{2} \mathrm{O}_{2}$ stimulation, and ERK was activated earlier than p38 MAPK and JNK activation. Additionally, the enhanced phosphorylation of JNK and p38 observed following stimulation with $\mathrm{H}_{2} \mathrm{O}_{2}$ was clearly attenuated by daphnetin pre-treatment in a dose-dependent manner. 
However, phosphorylation of ERK upon $\mathrm{H}_{2} \mathrm{O}_{2}$ stimulation was not attenuated by daphnetin treatment (Fig. 3). Furthermore, daphnetin was demonstrated to induce ERK activation, and ERK activation had no cumulative effect in PC12 cells stimulated with daphnetin and $\mathrm{H}_{2} \mathrm{O}_{2}$. Therefore, the present study hypothesized that ERK activation reached the maximum when PC12 cells were treated with daphnetin. These results suggest that daphnetin protects PC12 cells by suppressing p38 MAPK and JNK, and enhances ERK signaling.

HSP70 is induced in cells in response to a wide variety of chemical and physiological stresses, and its expression provides protection against cell death $(10,19,20)$. A previous study also suggested that HSP70 is protective in nervous system diseases (21), and HSP70 protects against neuronal, apoptosis at least in part, by inhibiting caspase-dependent and caspase-independent programmed cell-death pathways (22). Therefore, the present study investigated the effect of daphnetin on the expression of HSP70 in PC12 cells. The present data revealed that daphnetin clearly upregulates HSP70 expression in a dose- and time-dependent manner, which reached its maximum at $12 \mathrm{~h}$ (Fig. 4A and B).

To further determine the possible signal transduction pathway involved in daphnetin-induced HSP70 expression, the present study investigated pro-survival signaling protein activation, including Akt and ERK, upon daphnetin treatment. The activation of ERK was enhanced time-dependently upon daphnetin stimulation (Fig. 5A). By contrast, Akt phosphorylation did not exhibit a significant difference compared with unstimulated PC12 cells. Furthermore, a specific inhibitor of ERK revealed that ERK significantly suppressed HSP70 protein expression (Fig. 5B). These data confirmed that ERK, but not Akt, signaling is involved in daphnetin-mediated HSP70 expression.

In conclusion, the present study elucidated that daphnetin protects $\mathrm{PC} 12$ cells against $\mathrm{H}_{2} \mathrm{O}_{2}$-induced apoptosis by suppressing p38 MAPK and JNK signaling activation and increasing HSP70 expression via ERK signaling. Overall, the present results suggest that daphnetin may be a potential candidate for the treatment of neurodegenerative diseases. However, daphnetin should be further tested in animal models mimicking neurodegenerative diseases prior to being considered as a candidate for a clinical trial to prevent neurodegenerative disease progression in humans.

\section{Acknowledgements}

The present study was supported by the Natural Science Research Project of Anhui Colleges and Universities (grant no. KJ2016SD59), Outstanding Young Talent Support Program Key Projects in Anhui Colleges and Universities (grant no. gxyqZD2016173 ), National Nature Science Foundation of China (grant no. 31301171), Natural Science Research Project of Anhui Provincial Education Department (grant no. KJ2013B311) and Anhui Province Key Laboratory of Active Biological Macro-molecules (grant no. 1306C083008).

\section{References}

1. Gong QH, Shi XR, Hong ZY, Pan LL, Liu XH and Zhu YZ: A new hope for neurodegeneration: Possible role of hydrogen sulfide. J Alzheimers Dis 24 (Suppl 2): S173-S182, 2011.
2. Pan LL, Liu XH, Jia YL, Wu D, Xiong QH, Gong QH, Wang Y and Zhu YZ: A novel compound derived from danshensu inhibits apoptosis via upregulation of heme oxygenase-1 expression in SH-SY5Y cells. Biochim Biophys Acta 1830: 2861-2871, 2013.

3. Chong ZZ, Li F and Maiese K: Oxidative stress in the brain: Novel cellular targets that govern survival during neurodegenerative disease. Prog Neurobiol 75: 207-246, 2005.

4. Asaithambi A, Kanthasamy A, Saminathan H, Anantharam V and Kanthasamy AG: Protein kinase D1 (PKD1) activation mediates a compensatory protective response during early stages of oxidative stress-induced neuronal degeneration. Mol Neurodegener 6: 43, 2011.

5. Karuppagounder SS, Pinto JT, Xu H, Chen H-L, Beal MF and Gibson GE: Dietary supplementation with resveratrol reduces plaque pathology in a transgenic model of Alzheimer's disease. Neurochem Int 54: 111-118, 2009.

6. Yin W, Zhang X, Shi X and Li Y: Curcumin protects SH-SY5Y cells from oxidative stress by up-regulating $\mathrm{HO}-1$ via Phosphatidylinositol 3 Kinase/Akt/Nrf-2 and down-regulating HO-2. Mol Neurodegener 7 (Suppl 1): S14, 2012.

7. Zhang L, Ding W, Sun H, Zhou Q, Huang J, Li X, Xie Y and Chen J: Salidroside protects PC12 cells from $\mathrm{MPP}^{+}$-induced apoptosis via activation of the PI3K/Akt pathway. Food Chem Toxicol 50: 2591-2597, 2012.

8. Ouyang $\mathrm{X}$ and Yu T: Effect of Daphnetin on the expression of $\beta$-APP after the ischemia in the front brain of the mouse. Zhonghua Naoxueguanbing Zazhi 6: 2012 (In Chinese).

9. Kuang NZ, Fu YY, Gong SQ, Zeng ZP, Zhang ZQ and Chen ZY: Effects of Daphnetin extract on the expression of Bcl-2 and Bax mRNA during the apoptosis of SMMC-7721 cells. Guangdong Yiyao Zazhi 33: 1374-1377, 2012 (In Chinese).

10. Gao Y, Han C, Huang H, Xin Y, Xu Y, Luo L and Yin Z: Heat shock protein 70 together with its co-chaperone CHIP inhibits TNF-alpha induced apoptosis by promoting proteasomal degradation of apoptosis signal-regulating kinase1. Apoptosis 15: 822-833, 2010.

11. Sabirzhanov B, Stoica BA, Hanscom M, Piao CS and Faden AI: Over-expression of HSP70 attenuates caspase-dependent and caspase-independent pathways and inhibits neuronal apoptosis. J Neurochem 123: 542-554, 2012.

12. Chow AM, Tang DW, Hanif A and Brown IR: Induction of heat shock proteins in cerebral cortical cultures by celastrol. Cell Stress Chaperones 18: 155-160, 2013.

13. Kou X, Shen K, An Y, Qi S, Dai WX and Yin Z: Ampelopsin inhibits $\mathrm{H}_{2} \mathrm{O}_{2}$-induced apoptosis by ERK and Akt signaling pathways and up-regulation of heme oxygenase-1. Phytother Res 26: 988-994, 2012.

14. Wang Y, Li CF, Pan LM and Gao ZL: 7,8-Dihydroxycoumarin inhibits A549 human lung adenocarcinoma cell proliferation by inducing apoptosis via suppression of Akt/NF-kB signaling. Exp Ther Med 5: 1770-1774, 2013.

15. Du JS, Zhao Q, Zhang YL, Wang Y and Ma M: 7,8-dihydroxycoumarin may promote sciatic nerve regeneration by suppressing NF-kB expression in mice. Mol Med Rep 8: 1525-1530, 2013.

16. Du G, Tu H, Li X, Pei A, Chen J, Miao Z, Li J, Wang C, Xie H, $\mathrm{Xu} \mathrm{X}$ and Zhao H: Daphnetin, a natural coumarin derivative, provides the neuroprotection against glutamate-induced toxicity in HT22 cells and ischemic brain injury. Neurochem Res 39: 269-275, 2014.

17. Yang L, Yang Q, Zhang K, Li YJ, Wu YM, Liu SB, Zheng LH and Zhao MG: Neuroprotective effects of daphnetin against NMDA receptor-mediated excitotoxicity. Molecules 19: 14542-14555, 2014.

18. Yu S, Shen Y, Liu J and Ding F: Involvement of ERK1/2 pathway in neuroprotection by salidroside against hydrogen peroxide-induced apoptotic cell death. J Mol Neurosci 40: 321-331, 2010.

19. Takayama S, Reed JC and Homma S: Heat-shock proteins as regulators of apoptosis. Oncogene 22: 9041-9047, 2003.

20. Gao Y, Han C, Huang H, Xin Y, Xu Y, Luo L and Yin Z: Heat shock protein 70 together with its co-chaperone CHIP inhibits TNF-alpha induced apoptosis by promoting proteasomal degradation of apoptosis signal-regulating kinase1. Apoptosis 15: 822-833, 2010.

21. Turturici G, Sconzo G and Geraci F: Hsp70 and its molecular role in nervous system diseases. Biochem Res Int 2011: 618127, 2011.

22. Sabirzhanov B, Stoica BA, Hanscom M, Piao CS and Faden AI: Over-expression of HSP70 attenuates caspase-dependent and caspase-independent pathways and inhibits neuronal apoptosis. J Neurochem 123: 542-554, 2012. 